\title{
SUP-NORM ESTIMATES FOR BERGMAN-PROJECTIONS ON REGULATED DOMAINS
}

\author{
PAULA ERKKILÄ and JARI TASKINEN
}

\begin{abstract}
We give sufficient and necessary conditions for the boundedness of generalized Bergman projections on the space $L_{v}^{\infty}(\Omega)$. The conditions depend on the geometry of the simply connected domain $\Omega \subset \mathrm{C}$.
\end{abstract}

\section{Introduction}

We continue the study of Bergman type projections $P_{\varphi, \alpha, \eta}$ on $L_{v}^{p}(\Omega)$, where $\Omega \subset \mathrm{C}$ is a bounded, simply connected, so called regulated domain and $v$ is a weight on $\Omega$. In the papers [7] and [8] we found sufficient and necessary conditions for the boundedness of $P_{\varphi, \alpha, \eta}$ in terms of the geometry of $\Omega$. Those results are generalizations of the earlier works by Solovyov, [5], [6], and Békollé, [1]. However, only the cases $1<p<\infty$ were considered in [7] and [8]. In this paper we deal with the case $p=\infty$. For that we mention the reference [2], where projections on $L_{v}^{\infty}$ (D) were considered. However, these results are not formulated in terms of the geometry of $\Omega$.

We denote by $L_{v}^{\infty}(\Omega)$ and $H_{v}^{\infty}(\Omega)$ the spaces consisting of measurable, respectively, analytic, functions on $\Omega$ for which the norm

$$
\|f\|_{v}:=\underset{z \in \Omega}{\operatorname{ess} \sup } v(z)|f(z)|
$$

is finite. The weight function $v: \Omega \rightarrow \mathrm{R}$ is assumed strictly positive and continuous. If the weight is of the natural form, i.e. a power of the boundary distance,

$$
v(z):=(\operatorname{dist}(z, \partial \Omega))^{\sigma},
$$

we also denote by $L_{\sigma}^{\infty}(\Omega)$ the space $L_{v}^{\infty}(\Omega)$, and similarly in the analytic case. Since no bounded projection from $L^{\infty}(\mathrm{D})$ onto $H^{\infty}(\mathrm{D})$ exists, it only makes sense to assume $\sigma>0$. 
Our main result, Theorem 4.1, contains sufficient and necessary conditions for the boundedness of Bergman type projections $P_{\varphi, \alpha, \eta}$ on the space $L_{\sigma}^{\infty}(\Omega)$. (See Section 3 for basic properties of these projections. In general, all of them project onto subspaces of analytic functions.) The conditions involve several parameters connected with the projections and the geometry of the domain, and it is hard to find any simple characterization of boundedness of $P_{\varphi, \alpha, \eta}$. This is true even in very special cases: for example, denote by $P_{\varphi, \alpha, \alpha / 2}, \alpha>-1$, the orthogonal projection on $L_{\alpha}^{2}(\Omega)$ and consider the question of its boundedness on $L_{\sigma}^{\infty}(\Omega)$, and assume $\Omega$ has both inward and outward cusps. So we have $\delta_{1}=\pi=-\delta_{2}$ in Theorem 4.1. Boundedness holds for example, if

$$
4 \sigma-2>\alpha \geq 2 \sigma>0:
$$

then, in the assumptions of Theorem 4.1, we have $\eta:=\alpha / 2$ and also $1+$ $\eta-\sigma \geq 1$, hence (4.2) and the other assumptions are satisfied. For the same $\Omega$, the operator $P_{\varphi, \alpha, \alpha / 2}$ is bounded also in the case $\min (2 \sigma, 4 \sigma-2)>\alpha>$ $\max (2 \sigma-2, \sigma-1)$, since we then have $1>1+\eta-\sigma>0$, see Theorem 4.1.

The starting point of the proof is to transfer the situation from $\Omega$ to $D$ by using the Riemann map. Section 2 contains preliminary considerations on $D$ which correspond, again via the Riemann map, to the simplified case that $\Omega$ is a polyhedron.

We follow the notation and terminology of [7]. For properties of regulated domains we also refer to [4]. Let us shortly recall the definition. The regulated domain $\Omega \subset \mathrm{C}$ is simply connected and bounded and has a locally connected boundary. In this case a Riemann conformal map $\psi: D \rightarrow \Omega$ has a continuous extension to $\overline{\mathrm{D}}$ (still denoted by $\psi$ ). We define the curve $w(t)=\psi\left(e^{i t}\right)$, $0 \leq t \leq 2 \pi$. A crucial assumption is that each point of $\partial \Omega$ is attained only finitely often by $\psi$, and moreover,

$$
\beta(t):=\lim _{\tau \rightarrow t^{+}} \arg (w(\tau)-w(t))
$$

exists for all $t$ and defines a regulated function; the function $\beta$ is by definition regulated, if it can be approximated uniformly by step functions, i.e. for every $\varepsilon>0$ there exist $0=t_{0}<t_{1}<\cdots<t_{n}=2 \pi$ and constants $\gamma_{1}, \ldots, \gamma_{n}$ such that

$$
\left|\beta(t)-\gamma_{j}\right|<\varepsilon \quad \text { for } \quad t_{j-1}<t<t_{j}, j=1, \ldots, n .
$$

Geometrically, $\beta$ is the direction angle of the forward tangent of $\partial \Omega$ at $w(t)$. For more details, see [4], Section 3.5.

Given a regulated domain $\Omega \subset \mathrm{C}$, we fix some Riemann conformal map $\psi: \mathrm{D} \rightarrow \Omega$ and denote its inverse by $\varphi$. 
Recall that for $\Omega=\mathrm{D}$ the projection $P_{\alpha}:=P_{\mathrm{id}, \alpha, \alpha / 2}, \alpha>-1$, has the formula

$$
P_{\alpha} f(z)=(\alpha+1) \int_{\mathrm{D}} \frac{\left(1-|\zeta|^{2}\right)^{\alpha}}{(1-z \bar{\zeta})^{2+\alpha}} f(\zeta) d A(\zeta)
$$

\section{Preliminaries}

In this section we consider the boundedness of $P_{\alpha}$ in the case $\mathrm{D}$ for some rather simple, though non-radial weights $v$. They correspond, via the Riemann map, the case where the weight is as in (1.2) and $\Omega$ is diffeomorphic to a polyhedron, i.e., its boundary is $C^{1}$-smooth except for a finite number of corners. However, we only formulate the results for $D$ in this section.

The results are also used in the proof of the main theorem in Section 4.

Proposition 2.1. Let $\alpha>-1$ and $v(z):=(1-|z|)^{a}|1-z|^{b}$, where $a>\max (0,-b)$. The Bergman projection $P_{\alpha}: L_{v}^{\infty}(\mathrm{D}) \rightarrow L_{v}^{\infty}(\mathrm{D})$ is bounded, if and only if $\alpha>a+\max (b, 1)-2$.

The case $b=0$ follows from the usual Forelli-Rudin estimates, [9], Lemma 4.2.2. Concerning for example the sufficiency of the condition, if $f \in$ $L_{v}^{\infty}$ (D) with $|f(z)| \leq 1 / v(z)=(1-|z|)^{-a}$, and $\alpha>a-1$, then by ForelliRudin,

$$
\left|P_{\alpha} f(z)\right| \leq(\alpha+1) \int_{\mathrm{D}} \frac{(1-|\zeta|)^{\alpha-a}}{|1-z \bar{\zeta}|^{2+\alpha}} d A(\zeta) \leq \frac{C}{(1-|z|)^{a}} \quad \text { for } \quad z \in \mathrm{D} .
$$

So we assume in the following $b \neq 0$.

Since $|f(z)| \leq 1 / v(z)$ for every $f$ belonging to the closed unit ball of $L_{v}^{\infty}(\mathrm{D})$, the "if"-statement of Proposition 2.1 follows from the next lemma, taking $\lambda=0$.

Lemma 2.2. If $\alpha>a+\max (b, 1)-2, a>\max (0,-b)$ and $\lambda \in[0,2 \pi]$, then there exists a constant $C>0$ such that

$$
\begin{aligned}
\int_{\mathrm{D}} \frac{1}{(1-|\zeta|)^{a}\left|1-e^{-i \lambda} \zeta\right|^{b}} & \frac{(1-\mid \zeta)^{\alpha}}{|1-z \bar{\zeta}|^{2+\alpha}} d A(\zeta) \\
\leq & \frac{C}{(1-|z|)^{a}\left|1-e^{-i \lambda} z\right|^{b}} \quad \text { for } \quad z \in \mathrm{D}
\end{aligned}
$$

Proof of Lemma 2.2. By rotation symmetry, or a simple change of variable, it is enough to consider the case $\lambda=0$. 
Consider first the case $b>0$. Given $z \in \mathrm{D}$, we denote $\Omega_{1}:=\{\zeta \in \mathrm{D}$ | $|1-z| \geq 4|1-\zeta|\}$, and $\Omega_{2}:=\mathrm{D} \backslash \Omega_{1}$. If $\zeta \in \Omega_{1}$, then

$$
|1-z \bar{\zeta}|=|1-z+z(1-\bar{\zeta})| \geq|1-z|-|1-\zeta| \geq \frac{3}{4}|1-z| .
$$

We define $\beta:=\min (b, 1-\varepsilon)$, where $\varepsilon:=0$, if $b<1$, and $0<\varepsilon<1$ is chosen so small that $\alpha>a+b-2+\varepsilon$, if $b \geq 1$. As a consequence, $b-\beta \geq 0$, $0<\beta<1$, and $\alpha-a-b+\beta>-1$. Moreover, we write $\zeta=\varrho e^{i t}$ and note that the domain $\Omega_{1}$ is contained in the domain $\left\{\varrho e^{i t} \in \mathrm{D}|r:=1-| 1-z \mid / 4 \leq \varrho\right.$, $|t| \leq|1-z| / 2=: \theta\}$. We thus can estimate

$$
\begin{aligned}
\int_{\Omega_{1}} & \frac{1}{|1-\zeta|^{b}} \frac{(1-|\zeta|)^{\alpha-a}}{|1-z \bar{\zeta}|^{2+\alpha}} d A(\zeta) \\
& \leq \frac{C}{|1-z|^{2+\alpha}} \int_{\Omega_{1}} \frac{(1-|\zeta|)^{\alpha-a}}{|1-\zeta|^{\beta}|1-\zeta|^{b-\beta}} d A(\zeta) \\
& \leq \frac{C}{|1-z|^{2+\alpha}} \int_{\Omega_{1}} \frac{(1-|\zeta|)^{\alpha-a-b+\beta}}{|1-\zeta|^{\beta}} d A(\zeta) \\
& \leq \frac{C}{|1-z|^{2+\alpha}} \int_{r}^{1} \int_{-\theta}^{\theta} \frac{(1-\varrho)^{\alpha-a-b+\beta}}{\left(1+\varrho^{2}-2 \varrho \cos t\right)^{\beta / 2}} \varrho d \varrho d t
\end{aligned}
$$

We continue using the estimate $1+\varrho^{2}-2 \varrho \cos t \geq(1-\cos t) / 4 \geq t^{2} / 16$, and obtain for (2.4) the bound

$$
\begin{aligned}
& \frac{C}{|1-z|^{2+\alpha}} \int_{r}^{1}(1-\varrho)^{\alpha-a-b+\beta} d \varrho \int_{-\theta}^{\theta}|t|^{-\beta} d t \\
& \quad \leq \frac{C^{\prime}}{|1-z|^{2+\alpha}}\left[(1-\varrho)^{\alpha-a-b+\beta+1}\right]_{\varrho=r}^{1}\left[t^{-\beta+1}\right]_{t=0}^{\theta} \leq \frac{C^{\prime \prime}}{|1-z|^{a+b}}
\end{aligned}
$$

Moreover, by the Forelli-Rudin estimates, [9], Lemma 4.2.2,

$$
\begin{aligned}
\int_{\Omega_{2}} \frac{1}{|1-\zeta|^{b}} \frac{(1-|\zeta|)^{\alpha-a}}{|1-z \bar{\zeta}|^{2+\alpha}} d A(\zeta) & \leq \frac{C}{|1-z|^{b}} \int_{\Omega_{2}} \frac{(1-\mid \zeta)^{\alpha-a}}{|1-z \bar{\zeta}|^{2+\alpha}} d A(\zeta) \\
& \leq \frac{C}{|1-z|^{b}} \int_{\mathrm{D}} \frac{(1-|\zeta|)^{\alpha-a}}{|1-z \bar{\zeta}|^{2+\alpha}} d A(\zeta) \\
& \leq \frac{C^{\prime}}{(1-|z|)^{a}|1-z|^{b}}
\end{aligned}
$$


If $b<0$, we define $\Omega_{1}:=\{\zeta \in \mathrm{D}|| 1-z|\leq| 1-\zeta \mid / 2\}$, and $\Omega_{2}:=\mathrm{D} \backslash \Omega_{1}$. On $\Omega_{1}$ we use

$$
|1-z \bar{\zeta}|=|1-\bar{\zeta}+\bar{\zeta}(1-z)| \geq|1-\zeta|-|1-z| \geq \frac{1}{2}|1-\zeta| .
$$

Since $\alpha-a>-1$ we can use the Forelli-Rudin estimates to get

$$
\begin{aligned}
\int_{\Omega_{1}} \frac{1}{|1-\zeta|^{b}} \frac{(1-|\zeta|)^{\alpha-a}}{|1-z \bar{\zeta}|^{2+\alpha}} d A(\zeta) & \leq \int_{\Omega_{1}} \frac{(1-|\zeta|)^{\alpha-a}}{|1-z \bar{\zeta}|^{2+(\alpha-a)+(a+b)}} d A(\zeta) \\
& \leq \frac{C}{(1-|z|)^{a+b}} \leq \frac{C}{(1-|z|)^{a}|1-z|^{b}}
\end{aligned}
$$

On $\Omega_{2}$ we have $|1-z|^{-b} \geq C|1-\zeta|^{-b}$, so (2.6) can again be used for $\int_{\Omega_{2}}$.

Proof of Proposition 2.1. We need to consider the "only if"-statement. We prove the following fact, which is more than enough for our purposes: given $z \in \mathrm{D}$ and $M>4$, there exists a function $f \in L_{v}^{\infty}(\mathrm{D})$ with $\|f\|_{v}=1$ such that

$$
\left|P_{\alpha} f(z)\right| \geq C_{\alpha} M^{-\alpha+a+\max (b, 1)-2},
$$

if $\alpha<a+\max (b, 1)-2$, and $\left|P_{\alpha} f(z)\right| \geq C_{\alpha} \log M$, if $\alpha=a+\max (b, 1)-2$. Indeed, fixing a $z$, let us define

$$
f_{z, M}(\zeta):=e^{i \lambda(z, \zeta)}(1-|\zeta|)^{-a}|1-\zeta|^{-b}, \quad \text { if } \quad|\zeta| \leq 1-\frac{1}{M},
$$

where $\lambda(z, \zeta):=\arg \left((1-z \bar{\zeta})^{2+\alpha}\right)$, and $f_{z, M}(\zeta):=0$ otherwise. Then $\left\|f_{z, M}\right\|_{v}$ $=1$. Moreover, defining $\mathrm{D}_{M}:=\{\zeta \in \mathrm{D}|| \zeta \mid \leq 1-1 / M\}$,

$$
\begin{aligned}
P_{\alpha} f_{z, M}(z) & =C \int_{\mathrm{D}_{M}} \frac{\left(1-|\zeta|^{2}\right)^{\alpha}}{(1-z \bar{\zeta})^{2+\alpha}} \frac{e^{i \lambda(z, \zeta)}}{(1-|\zeta|)^{a}|1-\zeta|^{b}} d A(\zeta) \\
& \geq C^{\prime} \int_{\mathrm{D}_{M}} \frac{(1-|\zeta|)^{\alpha-a}}{|1-z \bar{\zeta}|^{2+\alpha}} \frac{1}{|1-\zeta|^{b}} d A(\zeta) \\
& \geq C^{\prime \prime} \int_{\mathrm{D}_{M}} \frac{\left(1-|\zeta|^{\alpha-a}\right.}{|1-\zeta|^{b}} d A(\zeta) .
\end{aligned}
$$

In the polar coordinates $\zeta=\varrho e^{i t}$ this can be estimated from below by a constant times

$$
\int_{0}^{2 \pi} \int_{1 / 2}^{1-1 / M} \frac{(1-\varrho)^{\alpha-a}}{\left(1+\varrho^{2}-2 \varrho \cos t\right)^{b / 2}} d \varrho d t
$$


If $b<1$, we bound this from below by

$$
C \int_{\pi / 2}^{3 \pi / 2} \int_{1 / 2}^{1-1 / M}(1-\varrho)^{\alpha-a} d \varrho d t=C^{\prime} \int_{1 / 2}^{1-1 / M}(1-\varrho)^{\alpha-a} d \varrho,
$$

and this clearly has the lower bound (2.9).

If $b \geq 1$, we estimate (2.12) from below by restricting $t$ to the interval $|t| \leq 1-\varrho$. Then, by the Taylor series, $\cos t \geq 1-t^{2} / 2 \geq 1-(1-\varrho)^{2} / 2$ and thus

$$
1+\varrho^{2}-2 \varrho \cos t \leq(1-\varrho)^{2}+\varrho(1-\varrho)^{2} \leq 2(1-\varrho)^{2},
$$

and for (2.12) we get the lower bound

$$
\begin{gathered}
C \int_{1 / 2}^{1-1 / M} \int_{0}^{1-\varrho} \frac{(1-\varrho)^{\alpha-a}}{\left(1+\varrho^{2}-2 \varrho \cos t\right)^{b / 2}} d \varrho d t \\
\quad \geq C^{\prime} \int_{1 / 2}^{1-1 / M} \int_{0}^{1-\varrho}(1-\varrho)^{\alpha-a-b} d \varrho d t \\
\quad \leq C^{\prime} \int_{1 / 2}^{1-1 / M}(1-\varrho)^{\alpha-a-b+1} d \varrho .
\end{gathered}
$$

Again this has the lower bound (2.9).

We want to generalize the above proposition. To that end we need another estimate.

Lemma 2.3. Let $\lambda \in[0,2 \pi], 0<\delta<1, \alpha>-1, a>0, \beta \in \mathrm{R}$ and $\alpha>a+\max (1, \beta)-2$. Define $\Omega_{\delta}:=\{\zeta \in \mathrm{D}|| \arg (\zeta)-\lambda \mid \leq \delta\}$. Then

$$
\int_{\Omega_{\delta}} \frac{1}{\left|1-e^{-i \lambda} \zeta\right|^{\beta}} \frac{(1-\mid \zeta)^{\alpha-a}}{|1-z \bar{\zeta}|^{2+\alpha}} d A(\zeta) \leq C_{\delta}
$$

for all $z$ with $|\arg z-\lambda| \geq 2 \delta$.

Proof. By rotation symmetry we can assume $\lambda=0$. Moreover, by assumptions, $|\arg (z \bar{\zeta})| \geq \delta$, hence, $|1-z \bar{\zeta}| \geq \sin \delta>0$. Hence, (2.16) is bounded by a $\delta$-dependent constant times

$$
\int_{\Omega_{\delta}} \frac{(1-|\zeta|)^{\alpha-a}}{|1-\zeta|^{\beta}} d A(\zeta) \leq C \int_{\Omega_{\delta}} \frac{(1-|\zeta|)^{\alpha-a-\max (1, \beta)+1-\varepsilon}}{|1-\zeta|^{1-\varepsilon}} d A(\zeta)
$$


where $\varepsilon>0$ is so small that $\alpha-a-\max (1, \beta)+2 \geq 2 \varepsilon$. Hence, (2.17) is bounded by a constant times

$$
\begin{aligned}
\int_{\Omega_{\delta}}(1-|\zeta|)^{-1+\varepsilon} \mid 1 & -\left.\zeta\right|^{-1+\varepsilon} d A(\zeta) \\
& \leq C \int_{0}^{1}(1-\varrho)^{-1+\varepsilon} \int_{0}^{2 \pi}\left|1-\varrho e^{i t}\right|^{-1+\varepsilon} d t d \varrho \leq C .
\end{aligned}
$$

Corollary 2.4. Let $n \in \mathrm{N}$ and let the real numbers $a$ and $b_{j}, j=1, \ldots, n$, satisfy $a>\max _{j=1, \ldots, n}\left(0,-b_{j}\right)$. Let the $n$ different numbers $\theta_{j} \in[0,2 \pi]$ be given, and let $v$ be the weight

$$
v(z):=(1-|z|)^{a} \prod_{j=1}^{n}\left|1-e^{-i \theta_{j}} z\right|^{b_{j}} .
$$

The Bergman projection $P_{\alpha}: L_{v}^{\infty}(\mathrm{D}) \rightarrow L_{v}^{\infty}(\mathrm{D})$ is bounded, if and only if $\alpha>a+\max _{j=1, \ldots, n}\left(1, b_{j}\right)-2$.

REMARK 2.5. If the projection is bounded, we actually can find a constant $C>0$ such that

$$
\begin{aligned}
\int_{\mathrm{D}} \frac{1}{\prod_{j=1}^{n}\left|1-e^{-i \theta_{j}} \zeta\right|^{b_{j}}} \frac{(1-|\zeta|)^{\alpha-a}}{|1-z \bar{\zeta}|^{2+\alpha}} & d A(\zeta) \\
\leq & \frac{C}{(1-|z|)^{a} \prod_{j=1}^{n}\left|1-e^{-i \theta_{j}} z\right|^{b_{j}}}
\end{aligned}
$$

for all $z \in \mathrm{D}$.

Proof OF Corollary 2.4 and Remark 2.5. Let us define $\delta:=\frac{1}{8} \min _{j \neq k}\left(\mid \theta_{j}-\right.$ $\left.\theta_{k} \mid\right)$. For every $j=1, \ldots, n$ define

$$
\begin{aligned}
\Omega_{j}^{-} & :=\left\{\zeta \in \mathrm{D}|| \arg (\zeta)-\theta_{j} \mid \leq \delta / 2\right\}, \\
\Omega_{j} & :=\left\{\zeta \in \mathrm{D}|| \arg (\zeta)-\theta_{j} \mid \leq \delta\right\} \quad \text { and } \\
\Omega_{j}^{+} & :=\left\{\zeta \in \mathrm{D}|| \arg (\zeta)-\theta_{j} \mid \leq 2 \delta\right\}
\end{aligned}
$$

and set $\Omega_{0}:=\mathrm{D} \backslash \cup_{j=1}^{n} \Omega_{j}$. We have $\left|1-e^{i \theta_{j}} \zeta\right| \geq C_{\delta}>0$, if $\zeta \notin \Omega_{j}$. The following thus holds:

$$
\int_{\mathrm{D}} \frac{1}{(1-|\zeta|)^{a}} \frac{1}{\prod_{j=1}^{n}\left|1-e^{-i \theta_{j}} \zeta\right|^{b_{j}}} \frac{(1-|\zeta|)^{\alpha}}{|1-z \bar{\zeta}|^{2+\alpha}} d A(\zeta)
$$




$$
\begin{aligned}
\leq C & \int_{\Omega_{0}} \frac{1}{(1-|\zeta|)^{a}} \frac{(1-|\zeta|)^{\alpha}}{|1-z \bar{\zeta}|^{2+\alpha}} d A(\zeta) \\
& +C \sum_{j=1}^{n} \int_{\Omega_{j}} \frac{1}{(1-|\zeta|)^{a}\left|1-e^{-i \theta_{j}} \zeta\right|^{b_{j}}} \frac{(1-|\zeta|)^{\alpha}}{|1-z \bar{\zeta}|^{2+\alpha}} d A(\zeta)
\end{aligned}
$$

The integral

$$
\int_{\Omega_{j}} \frac{1}{(1-|\zeta|)^{a}\left|1-e^{-i \theta_{j}} \zeta\right|^{b_{j}}} \frac{(1-|\zeta|)^{\alpha}}{|1-z \bar{\zeta}|^{2+\alpha}} d A(\zeta)
$$

is bounded by a constant (by Lemma 2.3), if $z \notin \Omega_{j}^{+}$, and using Lemma 2.2, it has the bound

$$
\frac{C}{(1-|z|)^{a}\left|1-e^{-i \theta_{j}} z\right|^{b_{j}}} \quad \text { for all } \quad z \in \Omega_{j}^{+} .
$$

Moreover, the integral over $\Omega_{0}$ is bounded on every $\Omega_{j}^{-}$and has the bound $C /(1-|z|)^{a}$ on $\Omega_{0}^{-}:=\mathrm{D} \backslash \cup_{j=1}^{n} \Omega_{j}^{-}$. Altogether, (2.21) is bounded by a constant times

$$
\frac{1}{(1-|z|)^{a}} \frac{1}{\prod_{j=1}^{n}\left|1-e^{-i \theta_{j}} z\right|^{b_{j}}} .
$$

As for the "only if"-part of Corollary 2.4, there are two cases. If $\max _{j}\left(b_{j}\right)<$ 1 , we pick up a $\lambda \in\left[0,2 \pi\right.$ [ such that $\left|\lambda-\theta_{j}\right| \geq 2 \delta$ for some $\delta>0$, for all $j$, and we define, for all $M>4$, for some fixed $z \in \mathrm{D}$, (2.25)

$$
f_{z, M}(\zeta):=e^{i \lambda(z, \zeta)}(1-|\zeta|)^{-a}, \quad \text { if }|\zeta| \leq 1-\frac{1}{M} \text { and }|\arg \zeta-\lambda| \leq \delta
$$

where $\lambda(z, \zeta):=\arg \left((1-z \bar{\zeta})^{2+\alpha}\right)$, and $f_{z, M}(\zeta):=0$ otherwise. Deducing as in (2.11)-(2.13) with $b=0$, we obtain for $\left|P_{\alpha} f_{z, M}(z)\right|$ the lower bound as in (2.9) (with 1 replacing $\max (b, 1)$ ).

In the case $\max \left(b_{j}\right) \geq 1$ we take the $j$ corresponding the maximal $b_{j}$ and work as around (2.15). Instead of the $t$-interval $|t| \leq 1-\varrho$ we work with the interval $\left|t-\theta_{j}\right| \leq \min (1-\varrho, \delta)$, but the idea of the proof is the same. 


\section{Remarks on generalized Bergman projections}

Let a simply connected $\Omega$ and a Riemann map $\varphi: \Omega \rightarrow \mathrm{D}$ be given. In this section we recall the basic properties of the following Bergman type projections:

$$
P_{\varphi, \alpha, \eta} f(z):=(\alpha+1) \int_{\Omega} \frac{\varphi^{\prime}(z) \bar{\varphi}^{\prime}(\zeta)\left(1-|\varphi(\zeta)|^{2}\right)^{\alpha}}{(1-\varphi(z) \bar{\varphi}(\zeta))^{2+\alpha}}\left(\frac{\varphi^{\prime}(z)}{\varphi^{\prime}(\zeta)}\right)^{\eta} f(\zeta) d A(\zeta),
$$

where $f: \Omega \rightarrow \mathrm{C}, z \in \Omega, \alpha>-1$ and $\eta \geq 0$. These projections were introduced in [7], (4.7) (in the case $\eta \in Z$ Z), where it was observed that they reproduce analytic functions. We call $P_{\varphi, \alpha, \alpha / 2}\left(=P_{\alpha}\right.$ in the case $\left.\Omega=\mathrm{D}\right)$ the orthogonal projection - maybe here is some small abuse of language. Given a weight $v$ on $\Omega$ we denote by $L_{v}^{2}(\Omega)$ the space with the norm $\left(\int_{\Omega}|f|^{2} v d A\right)^{1 / 2}$ and the inner product $(f \mid g):=\int_{\Omega} f \bar{g} v d A$. The following is true:

Lemma 3.1. The projection $P_{\varphi, \alpha, \alpha / 2}$ is the orthogonal projection from $L_{v}^{2}(\Omega)$ onto its subspace of analytic functions. Here

$$
v(z):=\left(1-|\varphi(z)|^{2}\right)^{\alpha}\left|\varphi^{\prime}(z)\right|^{-\alpha} .
$$

Notice that by the Koebe distortion theorem, [4], Corollary 1.4, we have

$$
\frac{1}{C} v(z) \leq \operatorname{dist}(z, \Omega)^{\alpha} \leq C v(z) \quad \text { for all } \quad z \in \Omega .
$$

Hence $P_{\varphi, \alpha, \alpha / 2}$ is also a bounded projection from $L_{\alpha}^{2}(\Omega):=L_{\text {dist }(z, \Omega)^{\alpha}}^{2}(\Omega)$ onto its subspace of analytic functions, though not necessarily the orthogonal one in this space.

For the convenience of the reader we give the straightforward proof for Lemma 3.1, since we do not know a good reference. It suffices to show $\left(g \mid P_{\varphi, \alpha, \alpha / 2} f\right)=\left(P_{\varphi, \alpha, \alpha / 2} g \mid f\right)$ for all $f, g \in L_{v}^{2}(\Omega)$. We have

$$
\int_{\Omega} g(z) \overline{P_{\varphi, \alpha, \alpha / 2} f(z)} v(z) d A(z)
$$$$
=(\alpha+1) \int_{\Omega} g(z)\left(\int_{\Omega} \frac{\overline{\varphi^{\prime}(z)} \varphi^{\prime}(\zeta)\left(1-|\varphi(\zeta)|^{2}\right)^{\alpha}}{(1-\overline{\varphi(z)} \varphi(\zeta))^{2+\alpha}}\left(\overline{\overline{\varphi^{\prime}(z)}}\right)^{\alpha / 2} \overline{f(\zeta)} d A(\zeta)\right)
$$$$
\cdot\left(1-|\varphi(z)|^{2}\right)^{\alpha}\left|\varphi^{\prime}(z)\right|^{-\alpha} d A(z)
$$$$
=(\alpha+1) \int_{\Omega}\left(\int_{\Omega} \frac{\overline{\varphi^{\prime}(z)} \varphi^{\prime}(\zeta)\left(1-|\varphi(z)|^{2}\right)^{\alpha}}{(1-\overline{\varphi(z)} \varphi(\zeta))^{2+\alpha}}\left(\frac{\varphi^{\prime}(\zeta)}{\varphi^{\prime}(z)}\right)^{\alpha / 2} g(z) d A(z)\right)
$$ 


$$
\begin{aligned}
& \cdot \overline{f(\zeta)}\left(1-|\varphi(\zeta)|^{2}\right)^{\alpha}\left|\varphi^{\prime}(\zeta)\right|^{-\alpha} d A(\zeta) \\
= & \int_{\Omega}\left(P_{\varphi, \alpha, \alpha / 2} g\right) \bar{f} v d A .
\end{aligned}
$$

Another straightforward fact is the following:

Lemma 3.2. The projection $P_{\varphi, \alpha, \eta}$ is bounded on $L_{\sigma}^{\infty}(\Omega)$ if and only if $P_{\alpha}$ is bounded on $L_{w}^{\infty}(\mathrm{D})$, where $w(z)=(1-|z|)^{\sigma}\left|\psi^{\prime}(z)\right|^{\sigma-1-\eta}$ for $z \in \mathrm{D}$.

Proof. Denote $v(z):=\operatorname{dist}(z, \partial \Omega)^{\sigma}$ for $z \in \Omega$ and let $f \in L_{\sigma}^{\infty}(\Omega)$. Assuming $P_{\alpha}$ bounded on the disc we get

$$
\begin{aligned}
\sup _{z \in \Omega}\left|P_{\varphi, \alpha, \eta} f(z)\right| v(z) & \\
\leq & C \sup _{z \in \Omega}\left|\int_{\Omega} \frac{\left(1-|\varphi(\zeta)|^{2}\right)^{\alpha}}{(1-\varphi(z) \overline{\varphi(\zeta)})^{2+\alpha}} \frac{\overline{\varphi^{\prime}(\zeta)}}{\varphi^{\prime}(\zeta)^{\eta}} f(\zeta) d A(\zeta)\right| \\
& \cdot\left(1-|\varphi(z)|^{2}\right)^{\sigma}\left|\varphi^{\prime}(z)\right|^{1+\eta-\sigma} \\
= & \left.C \sup _{z \in \Omega}\left|\int_{\mathrm{D}} \frac{\left(1-|\zeta|^{2}\right)^{\alpha}}{(1-\varphi(z) \bar{\zeta})^{2+\alpha}} \frac{\psi^{\prime}(\zeta)^{\eta}}{\overline{\psi^{\prime}(\zeta)}} f(\psi(\zeta))\right| \psi^{\prime}(\zeta)\right|^{2} d A(\zeta) \mid \\
& \cdot\left(1-|\varphi(z)|^{2}\right)^{\sigma}\left|\varphi^{\prime}(z)\right|^{1+\eta-\sigma} \\
= & C \sup _{z \in \mathrm{D}}\left|\int_{\mathrm{D}} \frac{\left(1-|\zeta|^{2}\right)^{\alpha}}{(1-z \bar{\zeta})^{2+\alpha}} f(\psi(\zeta)) \psi^{\prime}(\zeta)^{1+\eta} d A(\zeta)\right| \\
\quad & \cdot\left(1-|z|^{2}\right)^{\sigma}\left|\psi^{\prime}(z)\right|^{\sigma-1-\eta} \\
= & C\left\|P_{\alpha}\left((f \circ \psi) \psi^{\prime 1+\eta}\right)\right\|_{w} \leq C^{\prime}\left\|(f \circ \psi) \psi^{\prime 1+\eta}\right\|_{w} \\
= & C^{\prime} \sup _{z \in \mathrm{D}}|f(\psi(z))|\left|\psi^{\prime}(z)\right|^{1+\eta}\left(1-|z|^{2}\right)^{\sigma}\left|\psi^{\prime}(z)\right|^{\sigma-1-\eta} \\
\leq & C^{\prime \prime} \sup _{z \in \Omega}|f(z)| v(z),
\end{aligned}
$$

where on the first and last rows we used the Koebe distortion theorem, see (3.3).

The other direction is proven in the same way. 


\section{Main result}

Let $\Omega$ be a bounded, regulated domain and let the projection $P_{\varphi, \alpha, \eta}$ (see (3.1)) be given. Recall that our indices satisfy $\alpha>-1, \sigma>0$ and $\eta \geq 0$. Moreover, we define

$$
\begin{gathered}
\pi \geq \delta_{1}:=\sup _{t} \lim _{\tau \rightarrow 0^{+}}(\beta(t+\tau)-\beta(t-\tau)) \geq 0 \quad \text { and } \\
-\pi \leq \delta_{2}:=\inf _{t} \lim _{\tau \rightarrow 0^{+}}(\beta(t+\tau)-\beta(t-\tau)) \leq 0 .
\end{gathered}
$$

Since $\beta$ is the direction angle of the forward tangent of $\partial \Omega$, the number $\pi-\delta_{1}$ is, roughly, the angle of the sharpest outward pointing corner of $\Omega$. The number $\pi+\delta_{2}$ has an analogous interpretation for inward pointing corners. See [7] for more details on this definition.

THEOREM 4.1. (i) Assume that $1+\eta-\sigma \geq 0$ and $\sigma\left(1+\frac{\left|\delta_{2}\right|}{\pi}\right)>(1+\eta) \frac{\left|\delta_{2}\right|}{\pi}$. The Bergman projection $P_{\varphi, \alpha, \eta}$ is a bounded projection from $L_{\sigma}^{\infty}(\Omega)$ onto $H_{\sigma}^{\infty}(\Omega)$, if

$$
\alpha>\sigma+\max \left\{(1+\eta-\sigma) \frac{\delta_{1}}{\pi}, 1\right\}-2
$$

Conversely, if

$$
\alpha<\sigma+\max \left\{(1+\eta-\sigma) \frac{\delta_{1}}{\pi}, 1\right\}-2 .
$$

then $P_{\varphi, \alpha, \eta}$ is not bounded on $L_{\sigma}^{\infty}(\Omega)$.

(ii) Assume that $1+\eta-\sigma \leq 0$ and $\sigma\left(1+\frac{\delta_{1}}{\pi}\right)>(1+\eta) \frac{\delta_{1}}{\pi}$.

Then the result of (i) holds with $\delta_{1} \geq 0$ replaced by $\delta_{2} \leq 0$.

In the case (i) (respectively, (ii)) the condition (4.2) means a restriction for outward (resp. inward) pointing corners and cusps in the boundary of $\Omega$.

Proof of Teorem 4.1. By Lemma 3.2, the boundedness properties of $P_{\varphi, \alpha, \eta}$ on $L_{\sigma}^{\infty}(\Omega)$ are the same as those of $P_{\alpha}$ on $L_{w}^{\infty}(\mathrm{D})$, where $w: \mathrm{D} \rightarrow \mathrm{R}^{+}$ is the weight

$$
w(z):=\left|\psi^{\prime}(z)\right|^{\sigma-1-\eta}(1-|z|)^{\sigma} .
$$

Let us consider the case (i) and assume that (4.2) holds. Following [7] we derive a representation for $\left|\psi^{\prime}\right|^{\sigma-1-\eta}$ which reveals its essential factors.

Let $\varepsilon$ be so small that at least $0<\varepsilon<\frac{1}{100} \min (1, \sigma)$ and

$$
\alpha>(1+20 \varepsilon)\left(\sigma+\max \left\{(1+\eta-\sigma) \frac{\delta_{1}}{\pi}, 1\right\}\right)-2
$$


and

$$
\sigma\left(1+\frac{\left|\delta_{2}\right|}{\pi}\right)>(1+\eta) \frac{\left|\delta_{2}\right|}{\pi}+\varepsilon
$$

According to Section 2 of [7], the function $\left|\psi^{\prime}\right|^{\sigma-1-\eta}$ has the representation

$$
\left|\psi^{\prime}\right|^{\sigma-1-\eta}=C \exp \left(-\frac{\sigma-1-\eta}{2 \pi} \operatorname{Im} \int_{0}^{2 \pi} \frac{e^{i t}+z}{e^{i t}-z}(\beta(t)-t) d t\right),
$$

where $\beta:[0,2 \pi] \rightarrow \mathrm{R}$ is a regulated function. There exist finitely many points $0=\theta_{0}<\theta_{1}<\cdots<\theta_{n}=2 \pi$ such that

$$
\left|\beta(t)-t-\gamma_{j}\right|<\varepsilon^{3}
$$

for $\theta_{j-1}<t<\theta_{j}$, for some real constants $\gamma_{j}, j=1, \ldots, n$. We denote by $\beta_{1}$ and $\beta_{2}$ the $2 \pi$-periodic extensions to $\mathrm{R}$ of the functions

$$
\beta_{1}=\sum_{j=1}^{n} \gamma_{j} \chi_{j}, \quad \beta_{2}=\beta-t-\beta_{1},
$$

where $\chi_{j}(t)=1$ for $\theta_{j-1}<t<\theta_{j}$ and zero elsewhere. Clearly, the modulus of $\beta_{2}$ is bounded by $\varepsilon^{3}$ (by possibly redefining the function $\beta$ on the set $\left\{\theta_{0}, \ldots, \theta_{n}\right\}$ of measure 0 ). By the choice of the points $\theta_{j}$ we have (with $\left.\gamma_{0}:=\gamma_{n}\right)$

(4.10) $\left|\delta_{1}-\max _{0 \leq j<n}\left(\gamma_{j+1}-\gamma_{j}\right)\right| \leq 2 \varepsilon^{3} \quad$ and $\quad\left|\delta_{2}-\min _{0 \leq j<n}\left(\gamma_{j+1}-\gamma_{j}\right)\right| \leq 2 \varepsilon^{3}$.

Let us define for $j=1,2$

$$
\tilde{v}_{j}(z):=\exp \left(-\frac{\sigma-1-\eta}{2 \pi} \operatorname{Im} \int_{0}^{2 \pi} \frac{e^{i t}+z}{e^{i t}-z} \beta_{j}(t) d t\right) ;
$$

we thus have $\left|\psi^{\prime}\right|^{\sigma-1-\eta}=C \tilde{v}_{1} \tilde{v}_{2}$ and $w=C(1-|z|)^{\sigma} \tilde{v}_{1} \tilde{v}_{2}$. We still define $\rho:=\varepsilon /(10-\varepsilon)$ (hence $1+1 / \rho=10 / \varepsilon)$ and define the weights

$$
\nu_{1}:=\left((1-|z|)^{\sigma-\varepsilon^{2}} \tilde{v}_{1}\right)^{1+\rho}, \quad \nu_{2}:=\left((1-|z|)^{\varepsilon^{2}} \tilde{\nu}_{2}\right)^{1+1 / \rho} .
$$

(Notice that we shall prove $\tilde{\nu}_{2}(z) \leq C(1-|z|)^{-C \varepsilon^{3}}$, see Lemma 4.2, hence, possibly by diminishing $\varepsilon$, we still have

$$
\left.v_{2}(z) \leq C(1-|z|)^{\varepsilon} .\right)
$$


We want to prove that

$$
\int_{\mathrm{D}} \frac{1}{v_{j}(\zeta)} K_{\alpha}(z, \zeta) d A(\zeta) \leq \frac{C}{v_{j}(z)}
$$

for both $j=1$ and $j=2$. Here $K_{\alpha}$ is the modulus of the integral kernel of $P_{\alpha}$, i.e. $K_{\alpha}(z, \zeta):=C\left(1-|\zeta|^{2}\right)^{\alpha}|1-z \bar{\zeta}|^{-2-\alpha}$. Our theorem follows from this, since the Hölder inequality then implies

$$
\begin{aligned}
& \int_{\mathrm{D}} \frac{K_{\alpha}(z, \zeta)}{w(\zeta)} d A(\zeta) \\
& =\int_{\mathrm{D}} \frac{C}{(1-|\zeta|)^{\sigma-\varepsilon^{2}+\varepsilon^{2}} \tilde{v}_{1}(\zeta) \tilde{v}_{2}(\zeta)} K_{\alpha}(z, \zeta)^{\frac{1}{1+\rho}+\frac{1}{1+1 / \rho}} d A(\zeta) \\
& \leq C\left(\int_{\mathrm{D}} \frac{K_{\alpha}(z, \zeta)}{\left((1-|\zeta|)^{\sigma-\varepsilon^{2}} \tilde{v}_{1}(\zeta)\right)^{1+\rho}} d A(\zeta)\right)^{\frac{1}{1+\rho}} \\
& \cdot\left(\int_{\mathrm{D}} \frac{K_{\alpha}(z, \zeta)}{\left((1-|\zeta|)^{\varepsilon^{2}} \tilde{\nu}_{2}(\zeta)\right)^{1+1 / \rho}} d A(\zeta)\right)^{\frac{1}{1+1 / \rho}} \\
& =C\left(\int_{\mathrm{D}} \frac{K_{\alpha}(z, \zeta)}{v_{1}(\zeta)} d A(\zeta)\right)^{\frac{1}{1+\rho}}\left(\int_{\mathrm{D}} \frac{K_{\alpha}(z, \zeta)}{v_{2}(\zeta)} d A(\zeta)\right)^{\frac{1}{1+1 / \rho}} \\
& \leq C^{\prime}\left(\frac{1}{v_{1}(z)}\right)^{\frac{1}{1+\rho}}\left(\frac{1}{v_{2}(z)}\right)^{\frac{1}{1+1 / \rho}}=\frac{C^{\prime \prime}}{w(z)} \text {. }
\end{aligned}
$$

For $\tilde{v}_{1}$ one has the representation

$$
\tilde{v}_{1}(z)=\hat{v}_{1}(z) \prod_{j=0}^{n-1}\left|1-z e^{-i \theta_{j}}\right|^{-(\sigma-1-\eta)\left(\gamma_{j+1}-\gamma_{j}\right) / \pi}
$$

where $\hat{v}_{1}$ is a bounded function on $\mathrm{D}$ which is also bounded away from zero. One obtains (4.16) easily by taking the convolution of (4.9) times $-(\sigma-1-$ $\eta) /(2 \pi)$ with the conjugate Poisson kernel $-Q(t, z)$, see [3], p. 102. (The product stems from the principal part $2 r(\theta-t) /\left((1-r)^{2}+r(\theta-t)^{2}\right)$ of $-Q(t, z)$; here $z=r e^{i \theta}$. Use

$$
\int_{\theta_{j-1}}^{\theta_{j}} \frac{-2 r(\theta-t)}{(1-r)^{2}+r(\theta-t)^{2}} \gamma_{j} d t=\left[\gamma_{j} \log \left((1-r)^{2}+r(\theta-t)^{2}\right)\right]_{t=\theta_{j-1}}^{t=\theta_{j}}
$$


and

$$
\begin{aligned}
(1-r)^{2}+r\left(\theta-\theta_{j}\right)^{2} & \cong(1-r)^{2}+\left(\theta-\theta_{j}\right)^{2} \cong \operatorname{dist}\left(e^{i \theta}, r e^{i \theta_{j}}\right)^{2} \\
& \cong \operatorname{dist}\left(r e^{i \theta}, e^{i \theta_{j}}\right)^{2}=\left|z-e^{i \theta_{j}}\right|^{2}=\left|1-z e^{-i \theta_{j}}\right|^{2}
\end{aligned}
$$

A more careful calculation actually shows that $(4.16)$ holds with $\hat{v}_{1}=1$.) The inequality (4.14) is now obtained for $j=1$ from Remark 2.5, putting $a=(1+\rho)\left(\sigma-\varepsilon^{2}\right)$ and $b_{j}:=-(1+\rho)(\sigma-1-\eta)\left(\gamma_{j}-\gamma_{j-1}\right) / \pi$. That these numbers satisfy the required assumptions, follows from (4.5), (4.8), (4.10) and (4.12); the requirement $a>\max \left(0,-b_{j}\right)$ follows from (4.6), possibly by diminishing $\varepsilon$.

The proof of the boundedness of the projection is completed by the first statement of the following lemma:

LEMMA 4.2. The inequality (4.14) holds for $v_{2}$, and if $r<s<1,0 \leq \theta \leq$ $2 \pi$, then

$$
\frac{1}{C^{\prime}}\left(\frac{1-s}{1-r}\right)^{C \varepsilon^{3}} \leq \frac{\tilde{v}_{2}\left(r e^{i \theta}\right)}{\tilde{v}_{2}\left(s e^{i \theta}\right)} \leq C^{\prime}\left(\frac{1-r}{1-s}\right)^{C \varepsilon^{3}}
$$

This implies (4.13), since we obtain

$$
\tilde{v}_{2}(z) \leq C(1-|z|)^{-C \varepsilon^{3}}
$$

for all $z \in \mathrm{D}$.

We skip the proof of Lemma 4.2 for a moment, and instead we prove the unboundedness statement for the operator $P_{\varphi, \alpha, \eta}$; we again consider $P_{\alpha}$ on $L_{w}^{\infty}(\mathrm{D})$. So assume (4.3) holds. Denote by $k=\max (1, C,(1+\eta-\sigma) / \pi)$ where $C$ is the constant $C$ of (4.19) and choose $0<\varepsilon<\frac{1}{100} \min (1 / k, \sigma)$ so small that

$$
3 k \varepsilon+\alpha<\sigma+\max \left\{(1+\eta-\sigma) \frac{\delta_{1}}{\pi}, 1\right\}-2,
$$

and form the functions $\beta_{j}$ and weights $\tilde{v}_{j}, j=1,2$, as in (4.8)-(4.1) using the $\varepsilon$ of (4.21). (Notice that the statements of Lemma 4.2 still hold.) Let $m \in \mathbf{N}$ be such that $\gamma_{m+1}-\gamma_{m}=\max _{j}\left(\gamma_{j+1}-\gamma_{j}\right)=: \gamma$. Then (4.10) implies

$$
\max \left(1,(1+\eta-\sigma) \frac{\delta_{1}}{\pi}\right) \leq \max \left(1,(1+\eta-\sigma) \frac{\gamma}{\pi}\right)+2 k \varepsilon
$$

From now on we proceed as in the proof of Proposition 2.1. We fix a $z \in \mathrm{D}$ and let $M>4$ be arbitrary. If $(1+\eta-\sigma) \gamma / \pi<1$, we choose $0 \leq \lambda<2 \pi$ 
such that $\left|\lambda-\theta_{j}\right| \geq 2 \delta$ for all $j$, where $\delta:=\frac{1}{8} \min _{j \neq l}\left|\theta_{j}-\theta_{l}\right|$ and define

$$
f_{z, M}(\zeta):=\frac{e^{i \lambda(z, \zeta)}}{\left(1-|\zeta|^{2}\right)^{\sigma} \tilde{v}_{2}(\zeta)},
$$

if $|\zeta| \leq 1-1 / M$ and $|\arg \zeta-\lambda| \leq \delta$, and $f_{z, M}(\zeta)=0$ otherwise. Here $\lambda(z, \zeta):=\arg \left((1-z \bar{\zeta})^{2+\alpha}\right)$, and the choice of $\lambda$ implies that $f_{z, M} \in L_{w}^{\infty}(\mathrm{D})$ with $\left\|f_{z, M}\right\|_{w} \leq C$ (see (4.16) to get convinced that the factor $\tilde{\nu}_{1}$ of $w$ is essentially constant on the support of $f_{z, M}$ ).

If $(1+\eta-\sigma) \gamma / \pi \geq 1$, we define

$$
f_{z, M}(\zeta):=\frac{e^{i \lambda(z, \zeta)}}{\left(1-|\zeta|^{2}\right)^{\sigma}\left|1-\zeta e^{-i \theta_{m}}\right|^{(1+\eta-\sigma) \gamma / \pi} \tilde{\nu}_{2}(\zeta)},
$$

if $\zeta \in \mathrm{D}_{M, \delta}:=\left\{\zeta|| \zeta \mid \leq 1-1 / M\right.$ and $\left.\left|\arg \zeta-\theta_{m}\right| \leq \delta\right\}$, and $f_{z, M}(\zeta)=0$ otherwise. See above for the choice of the index $m$. In this case we get (since $\tilde{v}_{2}(\zeta) \leq C(1-|\zeta|)^{-k \varepsilon}$ by $\left.(4.20)\right)$

$\left|P_{\alpha} f_{z, M}(z)\right|$

$$
\geq C \int_{\mathrm{D}_{M, \delta}} \frac{(1-|\zeta|)^{\alpha}}{\left(1-|\zeta|^{2}\right)^{\sigma}\left|1-\zeta e^{-i \theta_{m}}\right|^{(1+\eta-\sigma) \gamma / \pi} \tilde{\nu}_{2}(\zeta)|1-z \bar{\zeta}|^{2+\alpha}} d A(\zeta)
$$

$$
\geq C^{\prime} \int_{\mathrm{D}_{M, \delta}} \frac{(1-|\zeta|)^{\alpha+k \varepsilon-\sigma}}{\left|1-\zeta e^{-i \theta_{m}}\right|^{(1+\eta-\sigma) \gamma / \pi}} d A(\zeta)
$$

But here we have, by (4.21) and (4.22),

$$
\begin{aligned}
\alpha<\sigma+\max \left(1,(1+\eta-\sigma) \frac{\delta_{1}}{\pi}\right) & -2-3 k \varepsilon \\
& \leq \sigma-k \varepsilon+(1+\eta-\sigma) \frac{\gamma}{\pi}-2,
\end{aligned}
$$

hence, using the same reasoning as in (2.11)-(2.15), we get for (4.25) the lower bound

$$
\left|P_{\alpha} f_{z, M}(z)\right| \geq C M^{-\alpha+\sigma-k \varepsilon+(1+\eta-\sigma) \gamma / \pi-2} .
$$

Since $M>0$ is arbitrary, this proves the unboundedness of the projection operator. The case (4.23) is treated in the same way.

It remains to prove Lemma 4.2. The proof is based on Lemma 1 of Section 3 of [2]. To obtain the inequality (4.14) one has to go through the proofs of [2]; the inequality is proven there, see (27) of [2].

First we remark that $v_{2}$ is found bounded, once (4.20) is proven, see (4.13); notice that in [2] the weights are assumed bounded. 
We first prove the bound (4.19) as follows.

$$
\begin{aligned}
& \text { (4.28) } \log \frac{\tilde{\nu}_{2}\left(r e^{i \theta}\right)}{\tilde{v}_{2}\left(s e^{i \theta}\right)} \leq\left|\log \tilde{v}_{2}\left(r e^{i \theta}\right)-\log \tilde{v}_{2}\left(s e^{i \theta}\right)\right| \\
& =\frac{|\sigma-1-\eta|}{2 \pi}\left|\int_{0}^{2 \pi} Q\left(t, r e^{i \theta}\right) \beta_{2}(t) d t-\int_{0}^{2 \pi} Q\left(t, s e^{i \theta}\right) \beta_{2}(t) d t\right| \\
& \leq C\left|\int_{0}^{2 \pi} Q\left(t, r e^{i \theta}\right) \beta_{2}(t) d t+\int_{|\theta-t|>1-r} \cot \left(\frac{\theta-t}{2}\right) \beta_{2}(t) d t\right| \\
& +C\left|\int_{|\theta-t|>1-r}-\cot \left(\frac{\theta-t}{2}\right) \beta_{2}(t) d t+\int_{|\theta-t|>1-s} \cot \left(\frac{\theta-t}{2}\right) \beta_{2}(t) d t\right| \\
& +C\left|\int_{|\theta-t|>1-s}-\cot \left(\frac{\theta-t}{2}\right) \beta_{2}(t) d t-\int_{0}^{2 \pi} Q\left(t, s e^{i \theta}\right) \beta_{2}(t) d t\right| .
\end{aligned}
$$

Denoting by $M f$ the Hardy-Littlewood maximal function of a function $f$ : $[0,2 \pi] \rightarrow R$, we get by [3], Section III, 1.2.,

$$
\begin{array}{r}
\left|\int_{0}^{2 \pi}-Q\left(t, r e^{i \theta}\right) \beta_{2}(t) d t-\int_{|\theta-t|>1-r} \cot \left(\frac{\theta-t}{2}\right) \beta_{2}(t) d t\right| \\
\leq C\left(M \beta_{2}\right)(\theta) \leq C^{\prime}\left\|\beta_{2}\right\|_{\infty} \leq C^{\prime \prime} \varepsilon^{3} .
\end{array}
$$

The last row has the same bound. On the second but last row we use $\mid \cot ((\theta-$ $t) / 2)-2 /(\theta-t) \mid \leq C$ and make a change of variable $t \mapsto \theta-t$, and so obtain for (4.28) the bound

$$
C \varepsilon^{3}+C\left\|\beta_{2}\right\|_{\infty} \int_{1-s \leq t \leq 1-r} \frac{1}{t} d t=C \varepsilon^{3}+C^{\prime} \varepsilon^{3} \log \left(\frac{1-r}{1-s}\right) .
$$

The second inequality of (4.19) follows. The first one can be proven in the same way.

Next we prove

$$
\frac{1}{\tilde{v}_{2}\left(e^{i \tau} z\right)} \leq C\left(\frac{|\tau|}{1-|z|}\right)^{C \varepsilon^{3}} \frac{1}{\tilde{v}_{2}(z)}
$$

for $z \in \mathrm{D},|\tau| \geq(1-|z|) / 2$. Assuming first $\tau>0$ and using the same method as above we obtain

(4.32) $\left|\log \tilde{v}_{2}(z)-\log \tilde{v}_{2}\left(e^{i \tau} z\right)\right|$ 


$$
\begin{aligned}
& =C\left|\int_{0}^{2 \pi} Q(t, z) \beta_{2}(t) d t-\int_{0}^{2 \pi} Q\left(t, e^{i \tau} z\right) \beta_{2}(t) d t\right| \\
& \leq C^{\prime} \varepsilon^{3}+C^{\prime} \mid \int_{|\theta-t|>1-r}-\cot \left(\frac{\theta-t}{2}\right) \beta_{2}(t) d t \\
& \quad+\int_{|\theta+\tau-t|>1-r} \cot \left(\frac{\theta+\tau-t}{2}\right) \beta_{2}(t) d t \mid \\
& \leq C^{\prime \prime} \varepsilon^{3}+C^{\prime}\left|\int_{|t|>1-r}-\frac{1}{t} \beta_{2}(t-\theta) d t+\int_{|t+\tau|>1-r} \frac{1}{t+\tau} \beta_{2}(t-\theta) d t\right|
\end{aligned}
$$

In the case $0 \leq \tau \leq 1-r$ the sum of the two integrals reads as

$$
\begin{aligned}
\int_{1-r-\tau}^{1-r} \frac{1}{t+\tau} \beta_{2}(t-\theta) d t & \\
& \quad+\int_{1-r}^{\pi}\left(\frac{1}{t+\tau}-\frac{1}{t}\right) \beta_{2}(t-\theta) d t+\int_{\pi-\tau}^{\pi}-\frac{1}{t} \beta_{2}(t-\theta) d t
\end{aligned}
$$

Estimating $\left|\beta_{2}\right|$ by $C \varepsilon^{3}$ all integrals can be seen to have the bound

$$
C \varepsilon^{3}+C \varepsilon^{3} \log \left(\frac{1-r+\tau}{1-r}\right) .
$$

The other cases are treated by same methods. The condition (4.2) follows.

We now turn to the Lemma 1, Section 3, of [2]. Given $n \in \mathrm{N}$ we denote $M_{n}:=\left\{1,2,3, \ldots, 2^{n}\right\}$ and $\lambda_{n, m}:=\left(1-2^{-n}\right) e^{2 \pi i m 2^{-n}}$ for $n \in \mathrm{N}$ and $m \in M_{n}$. We fix $N \in \mathrm{N}$, denote $M=2^{N} \in M_{N}$, and prove the following estimates:

$$
\begin{gathered}
\sum_{n<N} \sum_{m \in M_{n}} \frac{1}{m^{2+\alpha}} \frac{1}{v_{2}\left(\lambda_{n, m}\right)} \leq \frac{C}{v_{2}\left(\lambda_{N, M}\right)}, \\
\sum_{n \geq N} \sum_{m \leq 2^{n-N}} \frac{1}{v_{2}\left(\lambda_{n, m}\right)} 2^{(-n+N)(2+\alpha)} \leq \frac{C}{v_{2}\left(\lambda_{N, M}\right)}
\end{gathered}
$$

and

$$
\sum_{n \geq N} \sum_{2^{n-N} \leq m \leq 2^{n}} \frac{1}{m^{2+\alpha}} \frac{1}{v_{2}\left(\lambda_{n, m}\right)} \leq \frac{C}{v_{2}\left(\lambda_{N, M}\right)} .
$$

This is the special case of the assumption of Lemma 1, [2], where the points $\lambda_{N, M}$ are assumed to be on the real axis. It is enough to consider this case, since the general case can be obtained from this by a rotation. 
We have

$$
\frac{\tilde{\nu}_{2}\left(\lambda_{N, M}\right)}{\tilde{v}_{2}\left(\lambda_{n, m}\right)} \leq C 2^{C \varepsilon^{3}|N-n|} m^{C \varepsilon^{3}}
$$

Here the first factor $2^{C \varepsilon^{3}|N-n|}$ follows from (4.19), and the second from (4.2). Raising this to the power $1+1 / \varrho=10 / \varepsilon$ and taking into account the factor

$$
\left(1-\left|\lambda_{n, m}\right|\right)^{\varepsilon^{2}(10 / \varepsilon)}=2^{-10 \varepsilon n}
$$

of $v_{2}\left(\lambda_{n, m}\right)$ we obtain $(4.35)$ :

$$
\begin{aligned}
& \text { (4.40) } \sum_{n<N} \sum_{m \in M_{n}} \frac{1}{m^{2+\alpha}} \frac{1}{v_{2}\left(\lambda_{n, m}\right)} \\
& \leq C \sum_{n<N} \sum_{m \in M_{n}} \frac{1}{m^{2+\alpha}} \frac{2^{10 \varepsilon n}}{\tilde{v}_{2}\left(\lambda_{n, m}\right)^{10 / \varepsilon}} \\
& \leq C \sum_{n<N} \sum_{m \in M_{n}} \frac{1}{m^{2+\alpha-C 10 \varepsilon^{2}}} \frac{2^{10 \varepsilon n+C 10 \varepsilon^{2}|N-n|}}{\tilde{v}_{2}\left(\lambda_{N, M}\right)^{10 / \varepsilon}} \quad\left(\text { notice } 2+\alpha-C 10 \varepsilon^{2}>1\right) \\
& \leq C^{\prime} \sum_{n<N} 2^{10 \varepsilon N} 2^{\left(10 \varepsilon-C 10 \varepsilon^{2}\right)(n-N)} \frac{1}{\tilde{v}_{2}\left(\lambda_{N, M}\right)^{10 / \varepsilon}} \\
& \leq C_{\varepsilon} \frac{2^{10 \varepsilon N}}{\tilde{v}_{2}\left(\lambda_{N, M}\right)^{10 / \varepsilon}} \leq \frac{C_{\varepsilon}^{\prime}}{v_{2}\left(\lambda_{N, M}\right)} .
\end{aligned}
$$

As for (4.36),

$$
\begin{aligned}
\sum_{n \geq N} & \sum_{m \leq 2^{n-N}} \frac{1}{v_{2}\left(\lambda_{n, m}\right)} 2^{(-n+N)(2+\alpha)} \\
& \leq C \sum_{n \geq N} \sum_{m \leq 2^{n-N}} \frac{2^{10 \varepsilon n}}{\tilde{v}_{2}\left(\lambda_{n, m}\right)^{10 / \varepsilon}} 2^{(-n+N)(2+\alpha)} \\
& \leq C \sum_{n \geq N} \sum_{m \leq 2^{n-N}} \frac{m^{C \varepsilon^{2}}}{\tilde{v}_{2}\left(\lambda_{N, M}\right)^{10 / \varepsilon}} 2^{10 \varepsilon n+C 10 \varepsilon^{2}|N-n|+(-n+N)(2+\alpha)} \\
& \leq C^{\prime} \sum_{n \geq N} \frac{1}{\tilde{v}_{2}\left(\lambda_{N, M}\right)^{10 / \varepsilon}} 2^{\left(1+C 10 \varepsilon^{2}\right)(n-N)+10 \varepsilon n+C 10 \varepsilon^{2}|N-n|+(-n+N)(2+\alpha)}
\end{aligned}
$$




$$
=C^{\prime} \sum_{n \geq N} \frac{2^{10 \varepsilon N}}{\tilde{v}_{2}\left(\lambda_{N, M}\right)^{10 / \varepsilon}} 2^{(-n+N)\left(2+\alpha-1-C 20 \varepsilon^{2}-10 \varepsilon\right)}
$$

(4.41) $\leq C_{\varepsilon} \frac{2^{10 \varepsilon N}}{\tilde{v}_{2}\left(\lambda_{N, M}\right)^{10 / \varepsilon}} \leq \frac{C_{\varepsilon}^{\prime}}{v_{2}\left(\lambda_{N, M}\right)}$.

Finally, for (4.37) we estimate

$$
\begin{aligned}
& \sum_{n \geq N} \sum_{2^{n-N}<m \leq 2^{n}} \frac{1}{m^{2+\alpha}} \frac{1}{v_{2}\left(\lambda_{n, m}\right)} \\
& \leq C \sum_{n \geq N} \sum_{2^{n-N}<m \leq 2^{n}} \frac{1}{m^{2+\alpha}} \frac{2^{10 \varepsilon n}}{\tilde{v}_{2}\left(\lambda_{n, m}\right)^{10 / \varepsilon}} \\
& \leq C \sum_{n \geq N} \sum_{2^{n-N}<m \leq 2^{n}} \frac{1}{m^{2+\alpha-C 10 \varepsilon^{2}}} \frac{2^{10 \varepsilon n+C 10 \varepsilon^{2}|N-n|}}{\tilde{v}_{2}\left(\lambda_{N, M}\right)^{10 / \varepsilon}} \\
& \leq C \sum_{n \geq N} 2^{(-n+N)\left(2+\alpha-C 10 \varepsilon^{2}-1\right)} \frac{2^{10 \varepsilon n+C 10 \varepsilon^{2}|N-n|}}{\tilde{v}_{2}\left(\lambda_{N, M}\right)^{10 / \varepsilon}} \\
& \leq C \sum_{n \geq N} 2^{10 \varepsilon N} \frac{2^{(-n+N)\left(1+\alpha-20 C \varepsilon^{2}-10 \varepsilon\right)}}{\tilde{v}_{2}\left(\lambda_{N, M}\right)^{10 / \varepsilon}} \\
& \leq C \frac{2}{\varepsilon}_{\tilde{v}_{2}\left(\lambda_{N, M}\right)^{10 / \varepsilon}} \leq \frac{C_{\varepsilon}^{\prime}}{v_{2}\left(\lambda_{N, M}\right)} \cdot
\end{aligned}
$$

\section{REFERENCES}

1. Békollé, D., Projections sur des espaces de fonctions holomorphes dans des domains plans, Canad. J. Math. XXXVIII (1) (1986), 127-157.

2. Bonet, J., Engliš, M., Taskinen, J., Weighted $L^{\infty}$-estimates for Bergman projections, Studia Math. 171 (1) (2005), 67-92.

3. Garnett, J., Bounded Analytic Functions, Academic Press, New York, 1981.

4. Pommerenke, Ch., Boundary Behaviour of Conformal Maps, Grundlehren Math. Wiss. 299 (1992).

5. Solov'ev, A., Estimates in $L^{p}$ of the integral operators that are connected with spaces of analytic and harmonic functions, Soviet Math. Dokl. 19 (1978), 764-768.

6. Solov'ev, A., Estimates in $L^{p}$ of integral operators connected with spaces of analytic and harmonic functions, Siberian Math. J. 26 (1986), 440-460.

7. Taskinen, J., Regulated domains and Bergman type projections, Ann. Acad. Sci. Fenn. Math. 28 (2003), 55-68. 
8. Taskinen, J., Note on the paper "Regulated domains and Bergman type projections”, J. Funct. Spaces Appl. 2 (2004), 97-106.

9. Zhu, K., Operator Theory in Function Spaces, Marcel Decker, New York (1995).

\author{
UNIVERSITY OF HELSINKI \\ DEPARTMENT OF MATHEMATICS AND STATISTICS \\ P.O.BOX 68 \\ FIN-00014 HELSINKI \\ FINLAND \\ E-mail: taskinen@cc.helsinki.fi
}

